\title{
La formation à distance : dix principes inspirés par son histoire
}

\author{
Distance Education: Ten principles inspired by its history
}

\section{La formación a distancia: diez principios inspirados por su historia}

\author{
Sir John Daniel, Chancelier \\ Acsenda School of Management, Canada \\ odlsirjohn@gmail.com
}

RÉSUMÉ

L'article identifie une série de moments importants qui ont marqué les 2000 ans d'histoire de la formation à distance. II en déduit dix principes qui sont pertinents dans la période actuelle où, à la suite de la pandémie de COVID-19, la formation en ligne jouera un rôle plus significatif dans l'enseignement supérieur.

Mots-clés : documents pédagogiques, interaction, économies d'échelle, correspondance, rétroaction, Internet, télévision, radio, travail d'équipe, révolution industrielle

\section{ABSTRACT}

The paper picks out a series of important moments in the 2000-year history of distance education. It identifies ten principles relevant to the present situation when, following the COVID-19 pandemic, online learning will play a more significant role in higher education.

Keywords: learning materials, interaction, economies of scale, correspondence, feedback, Internet, television, radio, teamwork, industrial revolution 
El artículo identifica una serie de momentos clave que han marcado los 2000 años de historia de la formación a distancia. De ellos, se pueden deducir diez principios que resultan pertinentes en el período actual, en el que la formación a distancia debe desempeñar un papel más destacado todavía en la enseñanza superior debido a la pandemia Covid-19.

Palabras clave: materiales de aprendizaje, interacción, economías de escala, correspondencia, retroalimentación, internet, televisión, radio, trabajo en equipo, revolución industrial

\section{Introduction}

Beaucoup de gens supposent que la formation à distance a débuté vers l'an 2000 avec l'expansion rapide de l'Internet. D'ailleurs, l'utilisation du terme « enseignement en ligne » soutient l'idée que la formation à distance dépend étroitement de la disponibilité de l'Internet. En 2020, le recours massif à l'enseignement en ligne pour pallier les restrictions de la pandémie de COVID-19 n'a fait que renforcer la notion que la formation à distance est un phénomène nouveau.

Cet article prétend que les origines de la formation à distance remontent au moins au premier siècle de notre ère. En effet, le système d'enseignement créé pour les épitres de Saint Paul destinées aux premiers chrétiens, par le biais de leurs jeunes églises autour du bassin méditerranéen, comportait déjà deux éléments clés de l'apprentissage à distance : la distribution de matériel pédagogique et la constitution de groupes d'étude et de discussion autour des textes.

Au cours des siècles suivants, divers bouleversements sociaux et innovations technologiques ont fait évoluer ce simple système. D'abord, nous examinerons l'impact de l'invention de l'imprimerie par Gutenberg en 1440 sur la réforme protestante et l'encouragement de l'apprentissage indépendant. Ensuite, nous explorerons les éléments essentiels de la révolution industrielle du XVIII ${ }^{e}$ siècle tels que décrits par Adam Smith dans son œuvre classique La Richesse des Nations (1776/1881). Selon Smith, ces éléments sont la division du travail, l'utilisation de machines, la spécialisation et les économies d'échelle. Toutefois, ce n'est qu'au milieu du XX $X^{\circledR}$ siècle, avec la création d'universités à distance, appelées souvent "universités ouvertes ", que l'on a adopté ces pratiques dans l'enseignement supérieur afin d'offrir des programmes de qualité à des dizaines de milliers d'apprenants à distance.

Entre-temps, certains produits de la révolution industrielle ont grandement aidé ceux qui voulaient instruire les apprenants indépendants. Par exemple, l'implantation des réseaux de chemins de fer a mené directement à la création de services postaux, permettant le développement d'entreprises d'enseignement par correspondance dans les secteurs public et privé.

Les premières universités à distance avaient surtout recours au matériel pédagogique imprimé, aux médias de masse (radio et télévision), aux services postaux et aux communications téléphoniques, mais au cours des dernières décennies, de nouvelles technologies d'information et de communication se sont succédé rapidement. L'Internet a rendu possibles la production et la distribution de documents pédagogiques à bas coût, tout en favorisant une rétroaction plus rapide entre apprenants et enseignants. Ces technologies ont permis aux campus traditionnels, fondés sur l'enseignement présentiel, d'expérimenter l'enseignement à distance, souvent appelé formation en ligne, du fait de son recours à 
I'Internet. Ces tentatives d'inscrire les moyens de la formation à distance dans les missions de ces institutions ont été largement renforcées avec l'explosion de la maladie COVID-19 à travers le monde en 2020. Ayant été obligées de fermer leurs campus en pleine année scolaire, les universités se sont tournées vers l'enseignement en ligne. Ne disposant que de peu de temps pour s'y préparer, les professeurs ont diffusé leurs cours magistraux par le biais de technologies vidéo (comme Zoom) conçues pour l'organisation de réunions à distance (Daniel, 2020a, 2020b).

Dans ce texte, nous examinons plus en détail ces différentes étapes dans l'évolution de la formation à distance afin d'identifier dix principes clés. La pandémie de COVID-19 a créé une situation paradoxale. D'un côté, elle a stimulé un essor remarquable dans l'utilisation de la formation à distance, car maintes institutions qui enseignaient en présentiel se sont tournées en urgence vers l'apprentissage en ligne. De l'autre côté, à cause même de la rapidité de la transition, les dirigeants et les enseignants n'ont pas eu le temps d'adopter toutes les pratiques nécessaires à la bonne réussite de cette approche pédagogique.

Or, il est probable que, même après la pandémie, beaucoup d'établissements d'enseignement supérieur continueront à conserver, voire améliorer, leur capacité à intervenir à distance, même si ce n'est que pour être prêts à d'éventuelles nouvelles crises en tous genres. Nous espérons que les principes de succès que nous identifierons leur seront utiles dans cette entreprise.

\section{Principe \#1. Les deux piliers : matériel pédagogique et interactions humaines}

L'expansion de l'Église chrétienne depuis le début de notre ère faisait appel à la formation à distance, comme cela a été constaté à propos des épitres de Saint Paul. Ces lettres, distribuées, comme nous l'avons dit, par les moyens de l'époque aux petites communautés de chrétiens autour du bassin méditerranéen, incitaient les leaders de ces communautés à réunir les fidèles pour présenter le message et en discuter. C'est ainsi que l'Église a pu se mettre d'accord graduellement sur ses doctrines de base, renforcées plus tard par les conciles comme celui de Nicée, réuni par l'empereur Constantin en 325.

Ce qui nous concerne ici, c'est l'émergence du principe fondamental de la formation à distance, à savoir la nécessité d'associer l'élaboration de documents pédagogiques fiables et des possibilités d'échanges pour les apprenants. En effet, même lorsque l'alphabétisation est bien répandue, seule une minorité de gens apprendront convenablement en étudiant le matériel pédagogique de façon exclusivement indépendante.

\section{Principe \#2. Un matériel pédagogique disponible aux individus}

Un autre développement qui a bouleversé les comportements humains face au savoir a été l'invention de l'imprimerie par Gutenberg en 1640. Avant cette date, on ne disposait que de documents copiés à la main. Même la plupart de ceux et celles qui savaient lire n'avaient pas les moyens d'acheter ces manuscrits. Mais graduellement, grâce à l'imprimerie, les documents importants, en premier lieu la Bible, devenaient accessibles aux gens ordinaires avec de considérables conséquences sociales. Les causes de la réforme protestante du $\mathrm{XVl} l^{\mathrm{e}}$ siècle étaient complexes et multiples, mais la disponibilité accrue de livres et de journaux en a été un facteur important. En ce qui nous concerne, l'invention de l'imprimerie fut le précurseur d'une série de développements qui, se prolongeant jusqu'à Internet, ont favorisé les études indépendantes. 


\section{Principe \#3. Intégration des concepts de la révolution industrielle}

La révolution industrielle, qui a commencé en Europe au milieu du XVIII siècle, a peu à peu transformé toutes les sociétés qu'elle a touchées, en commençant par leurs méthodes de production. L'économiste Adam Smith (1776/1881), dans un passage célèbre de son ouvrage déjà cité, La Richesse des Nations, donne l'exemple d'une usine d'épingles en Normandie pour illustrer les composantes essentielles des méthodes de production introduites par cette révolution (Deleyre, 1751; Peaucelle, 1999). II s'agit de la division du travail, de l'utilisation de machines, de la spécialisation et des économies d'échelle. Nous y reviendrons car, même si ces méthodes ont rapidement transformé les secteurs de l'industrie et du commerce, ce n'est que vers la fin du $\mathrm{XX} \mathrm{X}^{\mathrm{e}}$ siècle, avec la création d'universités ouvertes, qu'elles ont été introduites dans l'éducation.

\section{Principe \#4. Engagement à servir les gens ordinaires}

L'une des conséquences les plus significatives de la révolution industrielle fut le développement de réseaux de chemins de fer au cours du XIX ${ }^{\mathrm{e}}$ siècle. Le transport par train a permis l'établissement de systèmes de distribution fiables sur l'ensemble des territoires nationaux, rendant possible la création de systèmes postaux. Le milieu du XIX ${ }^{e}$ siècle a vu émerger trois innovations éducatives significatives.

Premièrement, le Britannique Isaac Pitman, qui avait développé son système de sténographie en 1837, a créé pour l'enseigner le premier cours par correspondance dans les années 1840. II profitait ainsi du système postal national implanté à la même époque, système qui, à son tour, dépendait des chemins de fer pour être efficace. II a ainsi pu créer un système d'apprentissage bidirectionnel. Les apprenants recevaient les documents pédagogiques à domicile grâce au système postal qui servait aussi aux étudiants pour retourner leurs devoirs à Pitman pour correction et rétroaction. L'introduction d'une boucle de rétroaction a été une avancée de première importance dans la formation à distance, car elle permettait d'augmenter massivement la proportion d'apprenants réussissant leurs cours. L'un des éléments clés de l'histoire de la formation à distance depuis cette date est tout simplement le raffinement des mécanismes de rétroaction afin de les rendre plus rapides et plus pertinents.

Deuxièmement, cette époque féconde en inventions a stimulé une avancée radicale pour l'enseignement traditionnel en salle de classe. II s'agit du développement du tableau noir. Dans son essai de 1841, The Blackboard in Primary Schools, Bumstead décrivait son inventeur comme " l'un des plus grands bienfaiteurs de l'humanité » (Avenia, 2012). Aujourd'hui, on en ferait sans doute une évaluation plus nuancée car, en favorisant l'efficacité de l'enseignement, le tableau noir a aussi contribué à la standardisation de l'éducation publique et à la généralisation de la mémorisation comme approche pédagogique.

Enfin, c'est aussi dans cette période que l'on a vu naître, en 1858, le système d'études externes de I'Université de Londres. Le romancier Charles Dickens a appelé ce système "l'université du peuple », car il visait « le jeune cordonnier étudiant dans son grenier » et permettait à tous, partout dans le monde, de s'inscrire aux examens de l'université. Lorsque ce système d'études externes a fêté son $150^{\mathrm{e}}$ anniversaire en 2008, il comptait cinq lauréats du Prix Nobel parmi ses anciens étudiants, y compris Nelson Mandela (Kenyon Jones, 2008). 


\section{Principe \#5. Conversations didactiques guidées}

Entre le milieu du $X I X^{e}$ siècle et celui du $X X^{e}$, la formation à distance se manifestait surtout sous forme de cours par correspondance, offerts pour la plupart par des organisations commerciales et destinés à ceux qui voulaient parfaire leur formation pour faire face aux exigences d'un monde en cours d'industrialisation. L'intérêt des établissements universitaires pour cette nouvelle approche était plutôt limité, car au $\mathrm{XVIII}$ e siècle le programme externe de l'Université de Londres n'offrait pas de cours par correspondance, visant plutôt à permettre l'accès aux examens sans proposer aux postulants des formations pour s'y préparer.

Toutefois, en 1889, I'Université Queen's en Ontario est devenue la première université en Amérique du Nord à offrir des cours par correspondance. Le programme s'adressait surtout aux enseignants, mais on y ajouta en 1914 des cours en études bancaires et comptabilité. Puisqu'à cette époque il n'y avait aucune université à l'ouest de Toronto, Queen's attirait des inscriptions de l'Ouest canadien. Pour mieux servir ses étudiants, elle a créé un réseau de centres d'examens à travers le pays, réseau qui, en 1931, comptait 197 centres allant de Saint-Jean de Terre-Neuve jusqu'à Victoria, en Colombie britannique.

C'est dans la deuxième moitié du $X X^{e}$ siècle que l'enseignement par correspondance a attiré l'attention des chercheurs. II était décrit par un scientifique très connu, le suédois Borje Holmberg (1983), dans une phrase devenue célèbre, comme " une conversation didactique guidée " (guided didactic conversation). Plus tard, dans une lettre à De Vries, Holmberg (2016) dit s'être aperçu que le terme « didactic » en anglais implique souvent un enseignement unidirectionnel, voire autoritaire. Or, dans cette expression, Holmberg a voulu dire le contraire, c'est-à-dire une conversation ouverte dans laquelle le tuteur fait preuve d'empathie pour les idées de l'étudiant.

À cette même période, le commerce des cours par correspondance a attiré aussi l'attention des gouvernements, surtout après la publication d'un article choc par Mitford (1970) qui a levé le voile sur les pratiques immorales de certaines entreprises. Ce texte a provoqué la règlementation de ce commerce dans plusieurs pays et la publication d'un guide de bonnes pratiques par l'UNESCO (Mitford, 1979).

\section{Principe \#6. Profiter des médias de masse}

Le XXe siècle a vu l'arrivée des médias de masse : la radio, le film et la télévision. Tout comme ce fut le cas pour le tableau noir en 1841 , bien des gens ont qualifié de «révolutionnaire " chacune de ces inventions, prédisant qu'elles allaient transformer l'éducation. Nous assistons d'ailleurs au même phénomène avec des technologies encore plus récentes, telles que l'enseignement programmé, I'informatique, Internet et les médias sociaux. Nous verrons que chacun de ces développements peut faire avancer la formation à distance - et parfois la faire régresser -, mais qu'il n'existe pas, et qu'il n'existera probablement jamais, de média magique capable de surmonter tous les défis.

Ceci dit, la radio éducative et la télévision éducative ont joué, et jouent toujours dans certains pays, un rôle important. Bien entendu, ce sont des médias unidirectionnels - "didactiques " dans le sens anglais que Holmberg n'avait pas saisi. Mais, dans la plupart des cas, ces émissions éducatives, un peu comme les épitres de Saint Paul, sont reçues en classe par des groupes d'élèves où elles servent à déclencher des discussions. 


\section{Principe \#7. Intégrer les composantes}

Les années 1960 bouillonnaient d'idées. La période d'austérité de la Seconde Guerre mondiale touchait à sa fin, les médias de masse étaient en plein essor et le désir de réduire les inégalités s'est traduit par une expansion rapide de l'accès aux études universitaires. Le concept d'une grande université à distance a émergé de ce creuset de changements. C'est le premier ministre britannique Harold Wilson qui, dans un discours en 1965, a intégré deux tendances lourdes, les médias de communication et la demande pour un accès plus large aux universités, en proposant la création d'une « University of the Air ».

Faisant de cette idée un projet personnel, Wilson a pu surmonter l'opposition venant des milieux politiques, des médias et de la fonction publique. Un comité de planification composé d'universitaires chevronnés a élaboré le concept, le renommant Open University (OU). Leur but était de moderniser radicalement la formation à distance en créant un système multimédia avec un réseau national de tuteurs. En 1969, la nouvelle institution a reçu sa charte royale. Les fonctionnaires du ministère de l'Éducation avaient proposé que l'on commence timidement avec un projet pilote de quelques centaines d'étudiants pour tester le concept, mais le premier recteur, Walter Perry, conscient que le succès de l'établissement dépendrait d'économies d'échelle, a opté pour une première cohorte de 25000 étudiants en 1971 et en a ajouté 15000 autres en 1972.

Exprimant son désir d'être ouverte à tous, la nouvelle université avait comme politique d'admission " premier arrivé, premier servi », sans tenir compte de la scolarité antérieure des candidats. Dans les cinquante années qui ont succédé à sa fondation, plus de deux millions d'étudiants ont suivi une gamme de formations, allant du module jusqu'au doctorat. Aujourd'hui, avec 170000 inscrits et 13 millions de visiteurs à OpenLearn, le site Web qui présente ses cours en ligne gratuits, I'OU a considérablement élargi l'accès aux études supérieures.

\section{Principe \#8. Travail en équipe}

Dès ses débuts, l'OU a été renommée pour la qualité à la fois intellectuelle et pédagogique de ses enseignements. Les autres universités pouvaient facilement en évaluer la rigueur et la pertinence, car des milliers de leurs professeurs sont devenus tuteurs de l'OU parallèlement à leur fonction principale. Selon le recteur Perry, la clé du succès de l'OU et son innovation la plus importante ont été le travail en équipe. À la différence des approches artisanales traditionnelles, dans lesquelles le professeur concevait et délivrait seul ses cours, I'OU créait des équipes pour chaque cours, permettant des échanges très riches sur son contenu et sa pédagogie au cours de son élaboration. Souvent, on désignait un membre de l'équipe pour veiller à la diversité des exemples cités ou à l'universalité du langage utilisé.

\section{Principe \#9. Économies d'échelle; qualité d'échelle}

Le succès de l'Open University démontre l'avantage, en formation à distance, de fonctionner en termes d'échelle afin d'atteindre trois buts : tout d'abord élargir l'accès aux études supérieures, ensuite réaliser des économies dans la production du matériel pédagogique et, enfin, investir dans les ressources nécessaires pour assurer la qualité de l'ensemble du système. Ceci constitue un défi pour les établissements désireux d'intervenir dans des territoires à faible population, car même si Internet a fortement diminué les coûts de production et de distribution du matériel, le travail d'équipe qui caractérise une institution comme l'OU demeure onéreux. 
Un premier élément de solution est d'élargir la notion d'équipe au-delà de l'institution en profitant des ressources éducatives libres (REL). S'inspirant du mouvement vers les logiciels libres des années 1990, le partage de ressources éducatives, parfois élaborées par des équipes pluri-institutionnelles, prend de l'élan depuis dix ans. Un deuxième élément serait de mettre en place des plateformes numériques communes accessibles à tous les établissements d'un même territoire désireux de proposer des formations à distance accompagnées.

\section{Principe \#10. Assurer l'efficacité et la qualité de la formation à distance après la pandémie}

La pandémie de COVID-19 a créé une situation paradoxale pour la formation à distance. D'une part, des millions d'élèves et d'étudiants ont été obligés de suivre leur formation en dehors des salles de classe. Les termes " apprentissage à distance » et " formation en ligne " sont devenus des expressions courantes. D'autre part, l'expérience des systèmes éducatifs dans la période de COVID-19 a démontré que la formation à distance n'est pas adaptée à l'enseignement primaire et ne l'est pas beaucoup non plus pour le secondaire. Bien qu'elle soit plus appropriée pour le niveau supérieur, peu d'étudiants se déclaraient pleinement satisfaits de l'enseignement vidéo qu'ils avaient reçu dans l'urgence.

Puisque beaucoup d'universités projettent d'intégrer une plus grande composante de formation à distance dans leurs actions après la pandémie, ou du moins de garder la possibilité de faire appel à ce mode enseignement en cas d'urgence, elles devraient s'organiser pour en assurer la qualité.

Nous espérons que les principes que nous avons énoncés seront utiles dans cette entreprise.

\section{Liste de références}

Avenia, T. (2012, 28 octobre). The History and Future of the Chalkboard. ETEC540: Text, Technologies - Community Weblog. https://blogs.ubc.ca/etec540sept12/2012/10/28/the-history-and-future-of-the-chalkboard/

Daniel, J. S. (2020a). Education and the COVID-19 Pandemic. Prospects, 49, 91-96. https://doi.org/10.1007/s11125-02009464-3

Daniel, J. S. (2020b). COVID-19: A Two-Week Transition from Campus to Online at the Acsenda School of Management, Canada. Journal of Learning for Development, 7(3), 271-285. https://j14d.org/index.php/ej|4d/article/view/459/512

Deleyre, A. (1751). Épingle. Dans Diderot et d'Alembert (dir.), Encyclopédie ou Dictionnaire raisonné des sciences, des arts et des métiers ( $1^{\text {re }}$ éd., tome 5, p. 804-808). https://tinyurl.com/f2ys4d9z

Holmberg, B. (1983). Guided didactic conversation in distance education. Dans D. Sewart, D. Keegan, and B. Holmberg (dir.), Distance Education: International Perspectives (p. 114-122). Croom Helm. http://www.c3l.unioldenburg.de/cde/support/readings/holm83.pdf

Holmberg, B. (2016). Thirty Years Later: A Discipline of Distance Education. International Journal of E-learning and Distance Education / La revue internationale de l'apprentissage en ligne et de l'enseignement à distance, 31(2). http://www.ijede.ca/index.php/jde/article/view/1001

Kenyon Jones, C. (2008). The People's University: 150 Years of the University of London and its External Students. University of London.

Mitford, J. L. (1970). Let Us Now Appraise Famous Authors. The Atlantic Monthly, 226, 45-54.

Mitford, J. L. (1979). Poison Penmanship: The Gentle Art of Muckraking. Knopf.

Peaucelle, J.-L. (1999). La Division du Travail : Adam Smith et les encyclopédistes observent la fabrication des épingles en Normandie. Gérer et Comprendre, (57), 36-51. http://www.annales.org/gc/1999/gc09-99/35-51.pdf

Smith, A. (1881). Recherches sur la nature et les causes de la richesse des nations (G. Garnier, trad.). Université du Québec à Chicoutimi (ouvrage original publié en 1776 sous le titre An Inquiry into the Nature and Causes of the Wealth of Nations, W. Strahan and t. Cadell). http://classiques.uqac.ca/classiques/Smith_adam/smith_adam.html 